\title{
An integrated approach to friction surfacing process optimisation
}

\author{
I. Voutchkov ${ }^{\mathrm{a}, *}$, B. Jaworski ${ }^{\mathrm{b}}$, V.I. Vitanov ${ }^{\mathrm{c}}$, G.M. Bedford ${ }^{\mathrm{b}}$ \\ ${ }^{a}$ CEDC, School of Engineering Sciences, University of Southampton, Highfield, Southampton SO17 1BJ, UK \\ ${ }^{\mathrm{b}}$ Department of Mechanical and Manufacturing Engineering, University of Portsmouth, Anglesea Road, Anglesea Building, \\ Portsmouth PO1 3DJ, UK \\ ${ }^{\mathrm{c}}$ Building 30, School of Industrial and Manufacturing Sciences, Cranfield University, Cranfield, Bedfordshire MK43 OAL, UK
}

Received 31 July 2000; accepted in revised form 1 March 2001

\begin{abstract}
This paper discusses the procedures for data collection, management and optimisation of the friction surfacing process. Experimental set-up and characteristics of measuring equipment are found to match the requirements for accurate and unbiased data signals. The main friction surfacing parameters are identified and the first stage of the optimisation process is achieved by visually assessing the coatings and introducing the substrate speed vs. force map. The optimum values from this first stage forms a region around the middle of a trapezium-shaped area whose borders are found experimentally. Data collected for the second stage were analysed using the least squares method which were applied to find the coefficients of a second order regression model. Advantages of applying artificial intelligence methods to friction surfacing modelling are also described and the higher accuracy achieved using neural networks demonstrated. (c) 2001 Elsevier Science B.V. All rights reserved.
\end{abstract}

Keywords: Friction surfacing; Optimisation; Neural networks

\section{Introduction}

Friction surfacing is a solid phase process which produces coatings with hot forged microstructures that are typically fine and homogeneous and soundly bonded to the substrate.

The process (Fig. 1) involves rotating a consumable rod of the coating material onto a substrate and developing, through the friction generated at the rubbing interface, sufficient heat to plastically deform the end of the coating rod (mechtrode ${ }^{\mathrm{TM}}$ ). By moving the substrate across the face of the rotating rod, a layer of mechtrode $^{\mathrm{TM}}$ material is deposited. The coating is extremely regular and flat, without the familiar meniscus section profile experienced with fusion welding meth-

\footnotetext{
* Corresponding author.

E-mail addresses: iiv@soton.ac.uk (I. Voutchkov),v.vitanov@cranfield.ac.uk (V.I. Vitanov).
}

ods. The thickness of the coated layer is typically between 0.5 and $3 \mathrm{~mm}$ depending on mechtrode diameter and material. The process itself is environmentally clean, with no fumes, spatter or high intensity light emissions as in laser-based coating methods. It is also energy efficient because the heat is generated and used exactly where it is needed.

The process is used to create coatings with desired surface properties such as wear resistant coatings. For example, Frictec Ltd. successfully uses the process to produce long-life industrial machine knives [1].

There are two limitations which have slowed the wider application of this technique - lack of accurate sensing devices for reliable real-time process monitoring and an adequate process model. These limiting factors are addressed in this paper.

Friction surfacing involves a number of process parameters which directly affect the coating quality. A valid mathematical or analytical description of the 


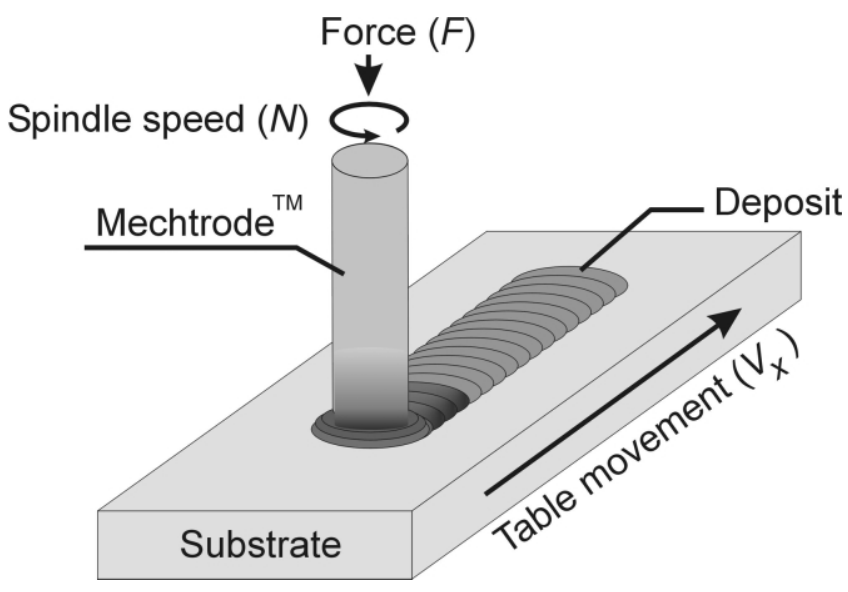

Fig. 1. Friction surfacing process.

process should successfully combine the significant parameters. The existing friction surfacing models [2-4] are based mainly on empirical equations or on experiments performed under a number of theoretical considerations. However, they do not account for the dynamics of the friction surfacing process parameters. The behaviour of these models has neither been analysed for cases when the friction surfacing model parameters vary as a response to different external influences, nor for measurement and estimation errors. Hence, the models have not been validated and their value for direct process monitoring and control is very limited.

The work described in this paper is concerned with the use of both mathematical and statistical approaches to collection, storage and analysis of process information as well as the development of useful process relationships.

\section{Experimental development and procedure}

\subsection{Parameter selection}

The friction surfacing machine consists of a power rotor which can move vertically with high precision (Z). Under the rotor there is an XY table, which can be positioned and moved accurately. The system is controlled using a serial computer link. Input parameters to the machine are the spindle rotation speed and direction $( \pm N)$, the $\mathrm{XY}$ table movement $\left(V_{x}\right)$ and the rod feed speed $\left(V_{z}\right)$ of the rotating Mechtrode ${ }^{\mathrm{TM}}$. For the experiments described in this paper, a piezoelectric washer, manufactured by Kistler Instruments, was used to measure the friction torque $\left(M_{s}\right)$ and the resultant friction force $(F)$. It was attached to the table directly under the substrate. The sensor has a measuring range for tensile force from 0 to $14000 \mathrm{~N}$ and $\pm 20000 \mathrm{~N} \mathrm{~cm}$ for torque. The sensitivity of the equipment is $\mathrm{pC} / \mathrm{N}$ -2.03 and $\mathrm{pC} / \mathrm{N} \mathrm{cm} 1.66$.

Temperature measurements of the mechtrode/coating/substrate interfaces are important to reveal the dynamics of heat and mass transfer during the process. Because of the nature of the process, measurements of

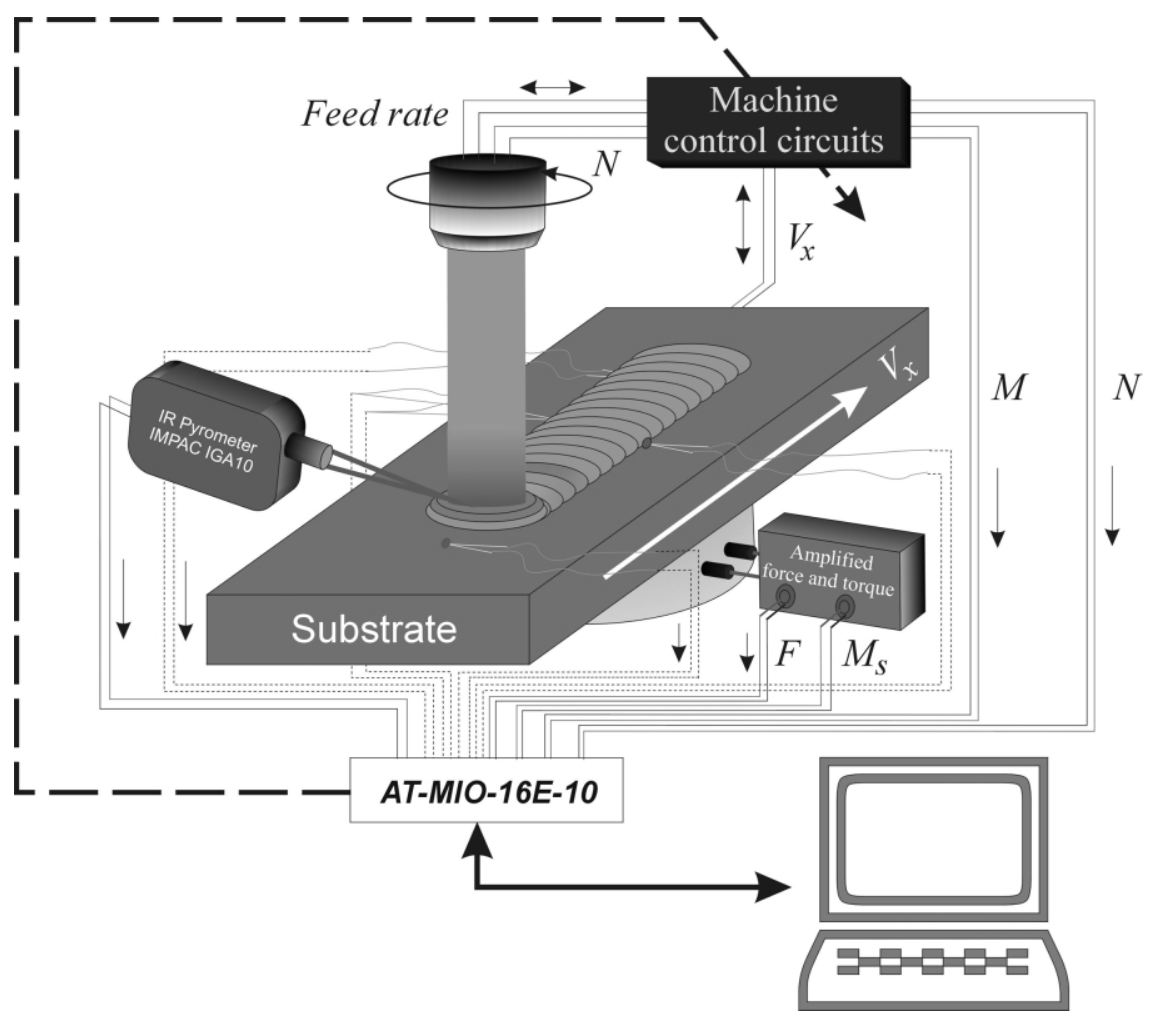

Fig. 2. Schematic diagram of the experimental set-up. 
the temperature profile at the surface of the mechtrode was acquired using a non-contact IR pyrometer, with an accuracy of $0.3 \%$, manufactured by IMPAC Electronic. The pyrometer has two options for the lenses, 80 and $250 \mathrm{~mm}$, with a spot size diameter of 0.3 and 0.5 $\mathrm{mm}$, respectively [5]. Temperature measurements were also carried out simultaneously, using up to $4 \mathrm{~K}$-type thermocouples spot-welded to the substrate.

Fig. 2 shows the experimental set-up. All observable parameters are measured and fed on-line to a multifunction I/O board (AT-MIO-16E-10) from National Instruments capable of data acquisition at a rate of 100 $\mathrm{kS} / \mathrm{sec}$. LabView application software has been used to automate the data acquisition process. This approach significantly reduces set-up time and running costs for friction surfacing experiments.

Because the friction surfacing machine is equipped with digital control, it was convenient to patch some of its circuits to the DAQ board. This enabled accurate information about mechtrode speed $\left(V_{z}\right)$, spindle speed $(N)$, traverse speed $\left(V_{x}\right)$ and spindle load $(M)$ to be relayed through the DAQ to the LabView based acquisition software. The DAQ board is capable of acquiring a maximum of 16 single-ended or 8 differential signals.

The system was successfully tested for up to 1000 samples per second for each channel. However, for most experiments, 100 samples per second have been found to be sufficient and producing a smaller data file.

Because all signals have dimensions of voltage, they are automatically scaled to proper physical dimensions at the end of the process cycle. They are also filtered to remove unwanted noise, leaving only the pure data signal for analysis. Several mathematical and statistical approaches were used to extract automatically the most useful values from the data file, pick and average values for all observed signals, duration of the steady process, ranges of variation and mean values, etc. This data was then recorded into a purpose-designed database, linked to Microsoft Access.

The friction surfacing machine is also equipped with a chamber in which experiments under different environments such as argon and water can be carried out.

Databases consist of numerous data sets, which visually are most commonly represented as rows. In a friction surfacing data set nine types of data are distinguished as follows.

1. Machine parameters are all variables related to the machine set-up-rod feedrate, spindle speed and traverse movement speed. Parameter data for the 4th axis, which is used to coat more complex substrate geometries, also belongs to this data set.

2. Environment parameters describe the environment in which the process takes place. Experiments in argon, air and water have been conducted. This data set indicates the gas/water flowrate and temperature of the environment.

3. Mechtrode parameters are values related to the consumable coating rod. Its material and diameter are the most important. Stickout and initial mechtrode length are used later to determine the rate of deposition, i.e. the amount of material transferred to the coating.

4. Substrate parameters represent information about substrate material, and geometry.

5. Temperature set-up identifies the positions in the $X$, $Y$ and $Z$ directions of all temperature sensors, including the infrared pyrometer. This data set also contains the operational parameters for the pyrometer, such as emissivity, lens, output range, etc.

6. Acquired values are those that come from the acquisition procedure, described earlier. It stores pick and average values for spindle speed, torque, force and temperatures.

7. Results section. Data about different characteristics and properties of the coating, such as width, thickness and bond strength.

8. Analysis section. Detailed metallographic examination.

9. Photo section. Photo images of each coating and associated metallography images are stored here.

\subsection{Data evaluation and optimisation procedures}

Some evaluation of coating characteristics can be carried out immediately after completion of a run. This is useful when experimental optimisation is being carried out. Decisions about future runs are taken following analysis of the actual data. The SIMPLEX method [6] for experimental optimisation was used to make decisions based on immediate estimation of the previous runs.

One of the immediate results is the appearance of the coating and this is evaluated visually and rated on a scale 0-10. Although it is not accurate enough for modelling purposes, it is very important for the first stage of optimisation, the so-called 'visual optimisation'. Evaluating coating appearance gives guidelines for finding the area of interest for more precise investigation. A proper looking run is a symptom of a stable process, which means that the heat and mass transfer balance conditions are achieved and all driving forces are in dynamic equilibrium. This process condition is sensitive to a number of parameters and should it be disturbed for any reason, the appearance of the coating suffers. The stability of friction surfacing is closely related to the quality of the product.

A new technique is used to store the images of the runs. The coatings are scanned directly, using an office scanner, rather than first taking a photograph and then 


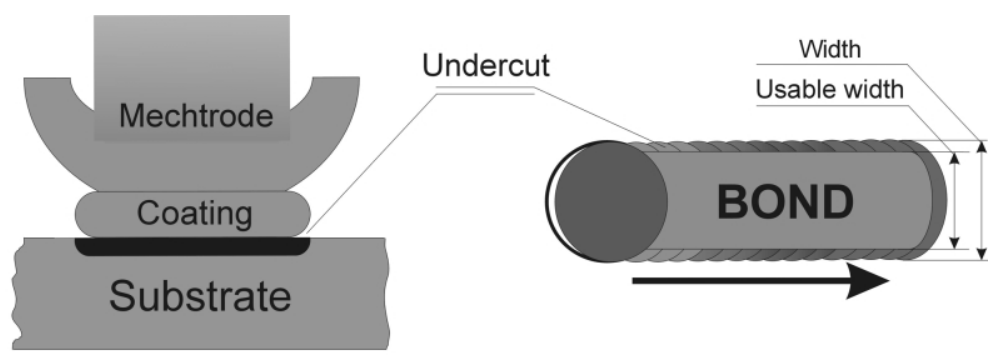

Fig. 3. Usable bond and undercut definition.

scanning it [7]. This enables higher resolution of the image, accurate and precise dimensioning, which later serves the purpose of detailed observation of run profile. Using image-processing software, the characteristic surface ripples of the coating can be automatically counted. This information is superimposed on the torque, force and temperature curves and a complete picture of the process is built up.

Using scanned runs, information about surface oxide colouring is also stored. Different oxide colours on the surface of the coating can be related to the rate of coating and heat transfer as well as to the rate of oxidation. It has been observed that certain colour patterns indicate good bond and this will be the subject of a further paper. During dynamically planned experiments this plays an important role. The runs are immediately evaluated by its surface oxide colouring and correct geometric appearance and the next experiment is planned accordingly.

To perform a more accurate analysis, the run can be scanned with a higher resolution (up to $1200 \mathrm{dpi}$ ) and the image can be processed and automatically assessed in less than $1 \mathrm{~min}$. Light microscopy was used to measure coating thickness and width on sectioned samples, and to evaluate the quality of the microstructural details of the mechtrode and coating.

As shown in Fig. 3, the usable bond width is always less than the width of the coating. There is invariably a small region at the edge of the coating that is not bonded, and this is called the undercut. The width of this narrow region (typically $<1 \mathrm{~mm}$ ) is closely related to the input machine parameters.

The comparative strength of the bond is currently measured using a simple push-off test, in which a hole was drilled from the back of the deposit, and a pin pushed through, while measuring the force. When the coating was detached from the substrate, a value was recorded. A $10-\mathrm{mm}$ length was cut from the substrate deposit using EDM. The hole diameter was $4 \mathrm{~mm}$ and positioned in the centre of the deposit cut-off. Because the width of the deposit was easily measured, it was possible to calculate the area on which the pressure was applied. Typical values were approximately 12 $\mathrm{kN} / \mathrm{cm}^{2}$. Currently, more sensitive and accurate strength tests are being developed. Detail of this procedure appears in Fig. 4.

\section{Results}

The results displayed in this section are for a Stainless Steel 304 rod diameter $5.00 \mathrm{~mm}$ length $50 \mathrm{~mm}$ deposited onto mild steel substrate. The substrate is machined and grinder finished. Spindle speed (N) during experiments was selected at $5500 \mathrm{rev} . / \mathrm{min}$. Substrates are $6 \mathrm{~mm}$ thick, for a $80 \times 45 \mathrm{~mm}$ area. It is held using a parallel vice, and mild steel spacers are placed underneath ensuring adequate positioning while simultaneously allowing strong forces to be applied. Runs are done across the substrates, and are usually $20 \times 5 \mathrm{~mm}$ wide. Achieved thickness varied from 0.3 up to $1.2 \mathrm{~mm}$. A variable length of the rod was used depending on process parameters. Pictures of runs can be seen in the Table 1 . That table indicates three typical images of friction surfacing coatings. It indicates front of the picture the evaluation that is given to the coating. That evaluation is used while performing the online first stage of the process optimisation. The subsequent runs are performed while simultaneously recording the signals from the sensors - such record can be seen in the Fig. 5. Using the collected pictures,

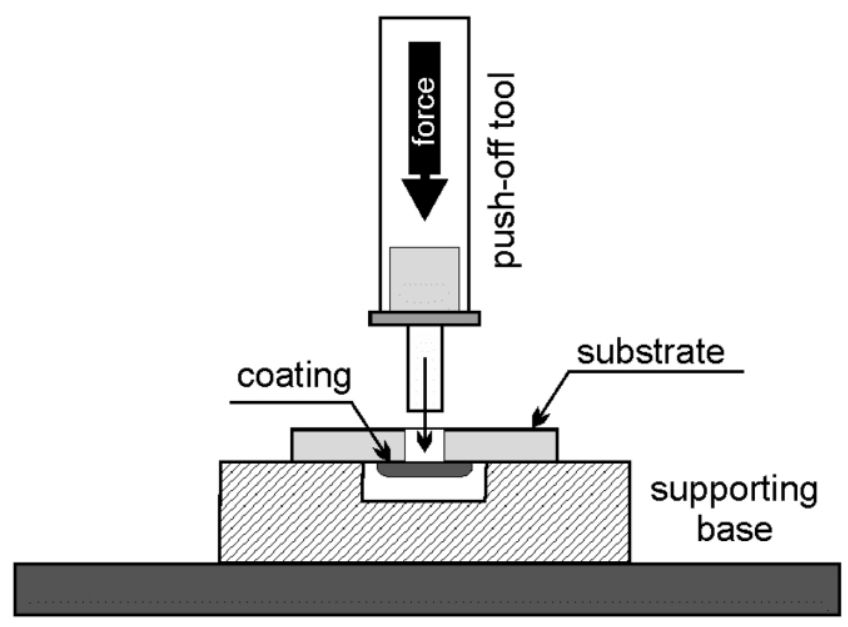

Fig. 4. Push off test definition. 


\begin{tabular}{|c|c|}
\hline Comment & Appearance \\
\hline $\begin{array}{c}\text { a) Good looking run; high level of } \\
\text { oxidation } \\
\text { Thick layer } \\
\text { Recommended appearance value }-5\end{array}$ & \\
\hline $\begin{array}{l}\text { b) Good looking run, low level of } \\
\text { oxidation } \\
\text { Additional colouring present } \\
\text { Recommended appearance value }-9\end{array}$ & \\
\hline $\begin{array}{l}\text { c) Poor looking run } \\
\text { Material has been splashed out, } \\
\text { rather than coated } \\
\text { Recommended appearance value }-1\end{array}$ & \\
\hline
\end{tabular}

a graph representing the appearance regards to the traverse speed $V_{x}$ and rod feed speed $V_{z}$ is plotted as in Fig. 6. The samples are then used for destructive bond test, from which results are plotted onto the Fig. 7.

\section{Discussion}

Friction surfacing is a complex process which depends on a number of parameters. For the purpose of modelling, optimisation and on-line control implementation, these process parameters can be grouped in the following way:

\subsection{Input}

\subsubsection{Controllable}

The values for the rotating rod speed $(N)$, the substrate speed $\left(V_{x}\right)$, and the rod feed speed $\left(V_{z}\right)$ are of greatest significance for successful surfacing.

\subsubsection{Non-controllable}

The coating bond strength and microstructural characteristics have a direct relation to the heat generated at the rubbing interface. The thermal system - substrate/coating/mechtrode - is also significant. Hence,

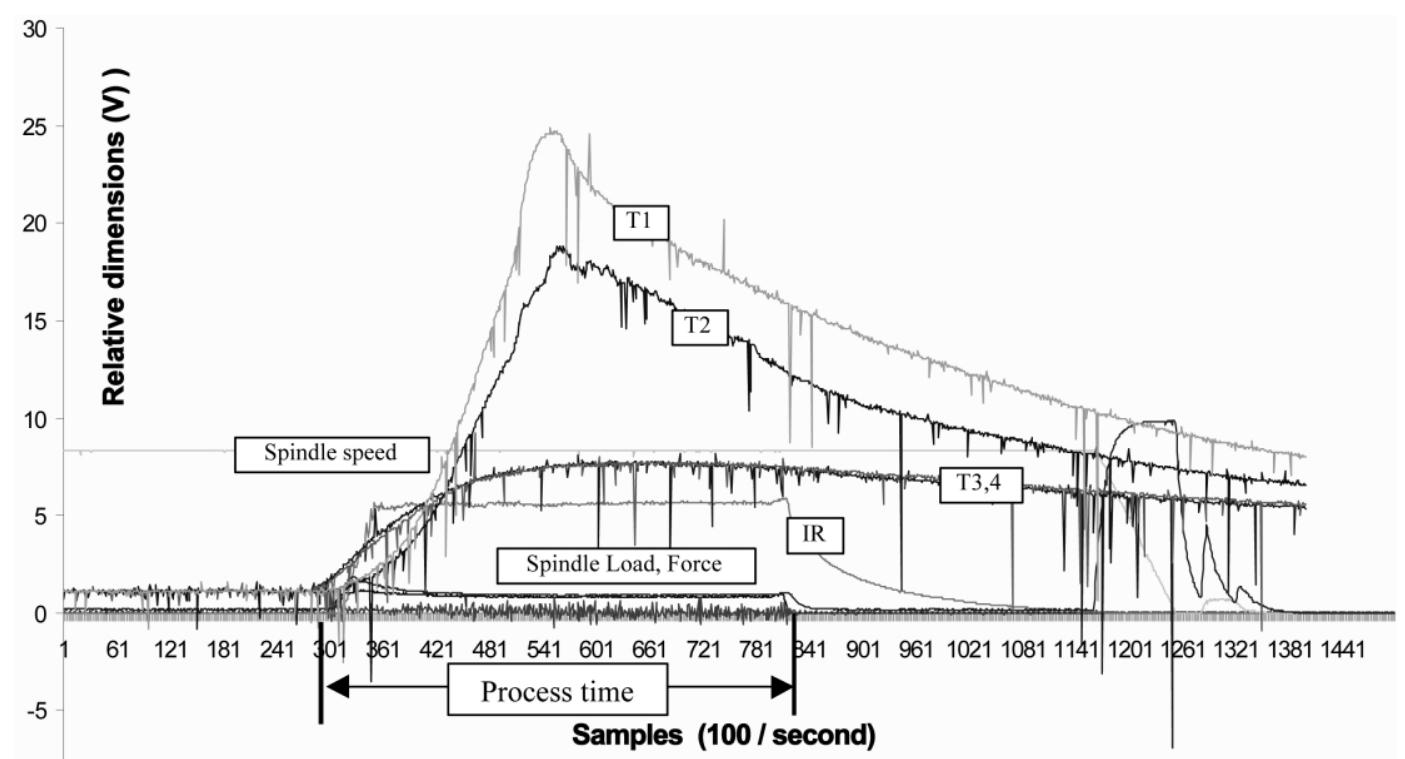

Fig. 5. Typical set of data, being recorded online from friction surfacing experiment. T1, T2, T3, T4 are signals from the thermocouples, IR is the temperature measured with the infrared pyrometer, spindle load and force come from the piezoelectric sensor. Spindle speed is patched from the machine control circuits. 


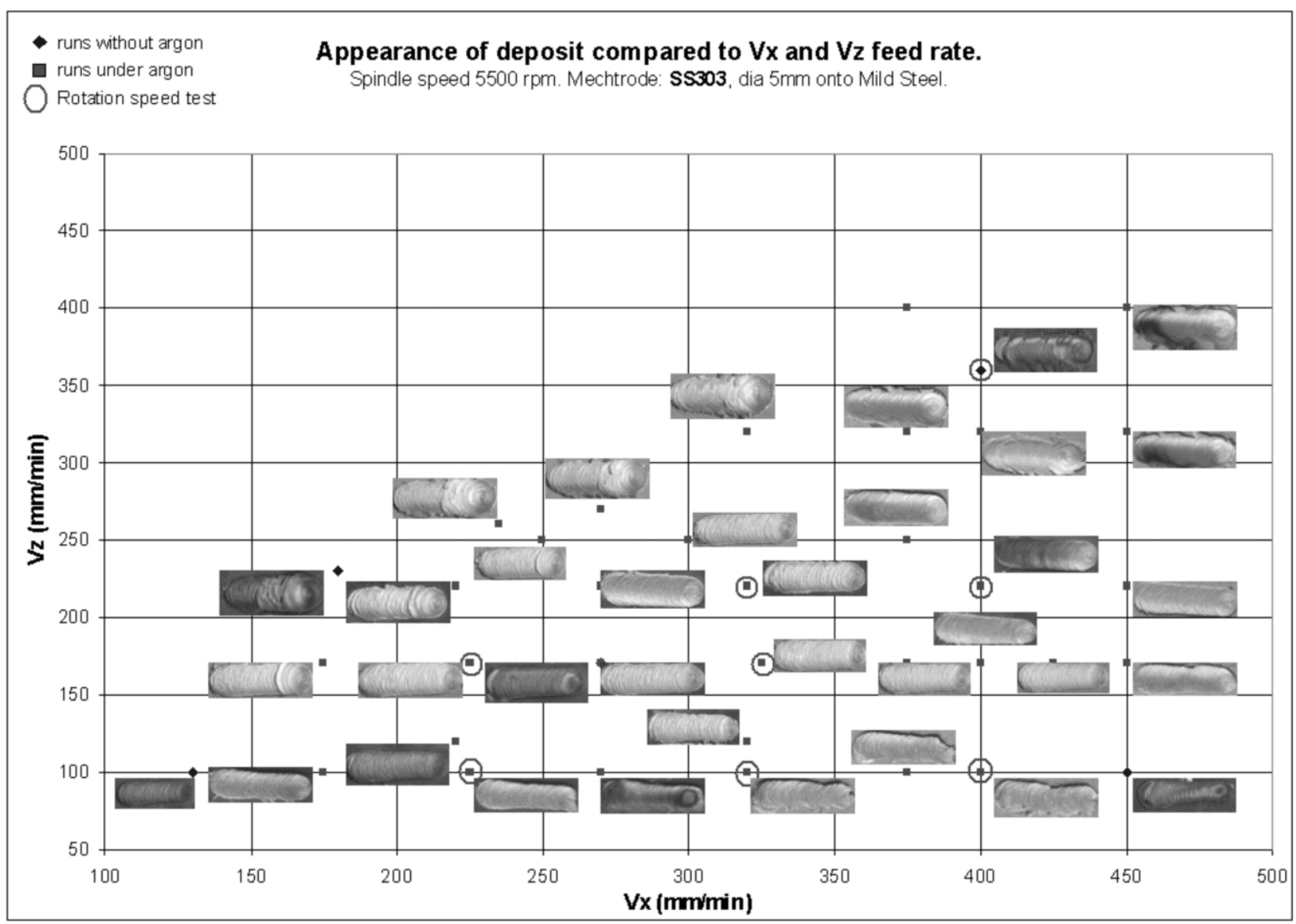

Fig. 6. $V_{x} / V_{z}$ response surface - first stage of friction surfacing optimisation $\mathrm{N}=5500 \mathrm{rev} . / \mathrm{min}$.

the substrate shape, size and material are another group of factors that influence the thermal system. These, together with the mechtrode diameter and material, form a group of factors which cannot be changed during the process.

\subsection{Output}

\subsubsection{On-line measurable}

Temperature at the rubbing interface $(T)$, friction torque $(M)$, applied force $(F)$ and roughness of the coating are just three of the output parameters which can be readily observed and measured.

\subsubsection{On-line non-measurable}

Bond width, bond strength and coating thickness are the remaining output parameters, which are often prescribed for given applications.

To successfully meet the requirements set by the non-measurable group of parameters in an on-line feedback system, they should be bounded in a functional relationship with the measurable parameters.

After the main parameters have been identified, the optimisation objective can be set to find the best set of input controllable parameters to achieve required coat- ing thickness, width and bond strength

Friction surfacing optimisation can be divided into three main stages:

1. Visual optimisation - getting the process to work for a given substrate and mechtrode.

2. Applying mathematical and statistical approaches to identify relationships between involved parameters.

3. Using optimisation techniques to extract the required values.

\subsection{Visual optimisation}

Stage 1 involves visual estimation of the appearance of run and measurement of coating thickness and width.

Depending on the different properties of the coating and substrate materials, the range of parameter values for which the process can work, varies for different coating material. Hence, before attempting any designed experimental procedure and detailed measurement these ranges have to be found. An analytical approach for this has been published recently [7].

Experimentally the appearance of the coating was 


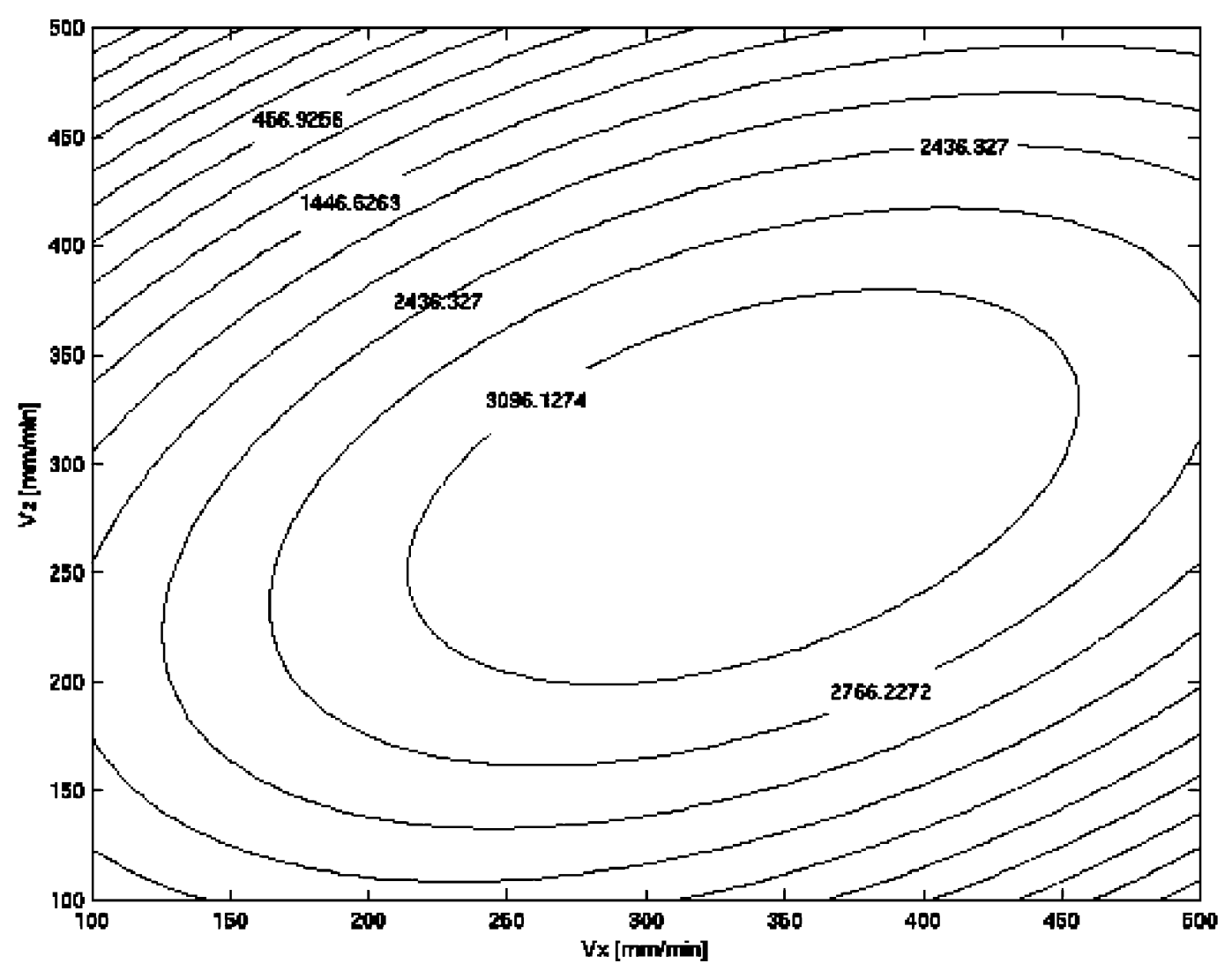

Fig. 7. $V_{x} / V_{z}$ bond strength response for a $\mathrm{SS} 304 \operatorname{rod} \varnothing 5$, onto a mild steel substrate.

used as the criteria for discarding the 'bad' runs and moving towards 'good' runs. Appearance is a complex criteria, based primarily on the observer's experience and judgement. At the end of the experiment, the run is given a number between 0 (for no coating achieved) and 10 (for ideal smooth and even coating).

Table 1 shows three runs, and their appearance evaluation number. These images were obtained using the scanning technique, described earlier. The table shows the runs carried out with stainless steel 304. Variation in $V_{x}$ and $F$, produced runs of different quality and appearance. A trapezium-shaped area is considered and as can be seen from Fig. 5, the coatings of good appearance, resulting from the process working correctly, are positioned within this area. In the lower left corner thick layer coatings, and a continuously narrowing run is produced. The upper left corner is described as a poor run where the coating material has been splashed out. According to experimental observations, the optimum appears to be around the centre of the trapezium diagonal.

\subsection{Application of mathematical and statistical approaches to identify relationships between involved parameters}

Using thickness, width and bond strength measurements, an attempt was made to derive a mathematical model using the Least Squares Method (LSM) [8]. A second order polynomial regression model was chosen to represent thickness, width and bond strength against the force and horizontal feedrate.

The regression coefficient $(r)$, was used as criteria for validation - the closer $r$ is to 1 , the better the model. On applying LSM to thickness, width and strength, values of $0.85,0.83$ and 0.56 , respectively, were calculated. Value for the strength model accuracy (0.56) is low, and cannot be used for optimisation purposes. An improvement to the thickness and width models is also required. A value for $r$ of 0.95 or more would indicate a satisfactory model.

The accuracy can be improved by changing the model structure to a higher order, or even by introducing other non-linear functions into it. An increase of order up to 5 th has been tried and the corresponding values for $r$ were $0.86,0.84$ and 0.58 , which does not satisfy the requirement for $r>0.95$

\subsection{Advantages of using neural networks}

Numerous friction surfacing experiments and discussions with process experts from Frictec, have revealed the following features of the process:

- The nature of the process is such that output parameters (such as bond width and bond strength) are difficult to measure on-line. 
- The measurement accuracy of some of the output parameters is insufficient, so that the conventional regression techniques cannot be applied, because the model structure (the regression functions and coefficients) are difficult to define.

- The bond quality (bond width and bond strength) depends on several controllable parameters such as mechtrode rotational speed, substrate movement, and mechtrode force.

- Factors which are not subject to control but are monitored only as part of the quality control sampling procedure. These include substrate size, substrate material, mechtrode material, which together define the thermal system for a given component.

- Selecting values for the processing parameters is currently based on expert knowledge.

A three layer neural network with two sigmoid and one linear layer, with 80 neurons in the hidden layer, was applied to approximate the function of thickness, width and bond strength with regard to $V_{x}$ and $F$. The data used to teach the network was the same as the ones used for the least squares method. The values for $r$ were $0.96,0.95$ and 0.75 . These values are significantly better than the ones calculated after the least squares method, although more improvement is needed. Applying fuzzy logic is believed to increase the accuracy further, since it can deal with a higher level of non-linearities and uncertainties [9].

The value of this work is in reducing the lead time and hence cost for determining the optimum parameters for a given coating material on a given substrate geometry. This is an important feature when developing the process for new applications because the optimal process parameters depend on the thermal system, which will vary when materials, mechtrode diameters and substrate geometries are changed.

The range of commercial applications currently includes the manufacture of machine knives for the food and pharmaceutical processing and packaging industries [10]. It is interesting to note that over the past decade no delamination has occurred from any of the thousands of knives manufactured by Frictec Limited using friction surfacing technology. This serves as a useful indicator of the bond quality and repeatability that can be achieved in the process. Furthermore, it indicates why such a high premium is placed on determining the optimum parameters which result in a repeatable process which is essential for commercial success. Other applications include hardfacing of valve seats with stellite, the repair and manufacture of parts for the gas turbine industry, notably gas turbine blades [11], and various types of tooling such as punches and drills.

\section{Conclusions}

1. The proposed experimental procedure and data collection routines are successful and can be confidently used for further friction surfacing studies.

2. The experimental set-up is suitable for automatic data collection.

3. The first stage of friction surfacing optimisation can be considered as accomplished. Optimum sets of parameters were found in respect to visual optimisation criteria.

4. The second stage of the optimisation process has been completed. Main operational parameters have been identified, as well as the optimisation objective. A mathematical model has been derived using conventional modelling methods, but it has been shown that the models need refining.

5. A Neural network model showed considerably better results over a conventional regression model.

\section{References}

[1] G.M. Bedford, P.J. Richards, Recent development in friction coating. Repair and reclamation, Royal Society, Sept. 1984, pp. 12.1-12.5.

[2] K. Fukakusa, Real rotational plane in friction heating process of similar materials with same diameter, IIW'98, Hamburg, Doc. No III-1113-98, (1998).

[3] K. Fukakusa, On fundamental characteristics of real rotation contact plane in friction surfacing. Fukui National College of Technology, IIW Doc., No III-1046-95, (1995).

[4] K. Fukakusa, On characteristics of real rotational contact plane. Fundamental study of friction surfacing, Q. J. JWS 14 (1) (1996) 2.

[5] R. Harvey, Neural Network Principles, Prentice-Hall, Englewood Cliffs, NJ, 1994.

[6] W.G. Spendley, G.R. Hext, F.R. Himsworth, The sequential application of simplex designs in optimization and evolutionary operation, Technometrics 4 (4) (1962).

[7] V.I. Vitanov, I.I. Voutchkov, G.M. Bedford, Decision support system to optimise the FRICTEC (Friction Surfacing) Process. Proceedings of the 15th International CAPE Conference CAPE'99, Durham, 19-21 April (1999), pp. 471-476.

[8] P. Whittle, Prediction and Regulation by Linear Least-Square Methods, English Universities Press, London, 1963.

[9] W. Pedrycz, A. Rocha, Fuzzy-set based models of neurons and knowledge-based networks, IEEE Trans. Fuzzy Syst. 1 (4) (1993).

[10] G.M. Bedford, Friction surfacing for wear application, Metals Mater. 6 (11) (1990) 702-705.

[11] G.M. Bedford, A. Davies, J.R. Sharp, Micro-friction surfacing in the manufacture and repair of gas turbine blades. Third International Charles Parsons Turbine Conference, Newcastle, April (1995), pp. 683-693. 